\title{
Caracterización espeleológica e inventario biológico de la Caverna del Diablo en el municipio de Becerril, Departamento del Cesar, Colombia
}

\section{Speleological characterization and biological inventory of the Diablo's Cavern in the municipality of Becerril, Cesar's Department, Colombia}

\section{Caracterização espeleológica e inventário biológico da Caverna do Diabo no município de Becerril, Estado do Cesar, Colômbia}

\author{
Frederick Vides-Navarro ${ }^{1}$, Ángelo Montes-Calderón ${ }^{2}$, Giovanni Fernández-Cuello ${ }^{3}$, Elías Rojas-Martínez ${ }^{4}$
}

Forma de citar: F. Vides-Navarro, A. Montes-Calderón, G. Fernández-Cuello, E. Rojas-Martínez, “Caracterización espeleológica e inventario biológico de la Caverna del Diablo en el municipio de Becerril, Departamento del Cesar, Colombia", Respuestas, vol. 20, no. 2, pp. 93-104, 2015.

Recibido:

Noviembre 13 de 2014

Aceptado: Marzo 30 de 2015

Ingeniería de Minas fvides06@gmail.com ORCID: 0000-0002-1987-6868 Fundación Universitaria del Área Andina

Valledupar-Colombia

${ }^{2}$ Ingeniería de Minas angelo.mc@hotmail.com ORCID: 0000-0002-2188-5237 Fundación Universitaria del Área Andina

Valledupar-Colombia

${ }^{3}$ Ingeniería de Minas (c) geovanny_fdz_2415@hotmail.com ORCID: 0000-0002-3704-9372 Fundación Universitaria del Área Andina Valledupar-Colombia

${ }^{4}$ Especialización en Minería a Cielo Abierto

erojas@areandina.edu.co ORCID: 0000-0003-0402-1565 Fundación Universitaria del Área Andina Valledupar-Colombia

\section{Resumen}

Objetivo: Este estudio tiene como objetivo caracterizar los aspectos más relevantes de la caverna del Diablo, entre los que se incluyen el componente biofísico, geológico, espeleológico y morfológico. Metodología: Se ha realizado una investigación descriptiva a partir de observaciones in situ, trabajos técnicos específicos de topografía, geología, espeleología y biología, acompañado de análisis de literatura relacionada. Resultados: Los métodos utilizados permitieron conocer la riqueza natural en el interior de la caverna, donde sobresalen geoformas kársticas (espeleotemas), presencia de peces, crustáceos, y otros organismos que podrían ser endémicos. La información obtenida indica que el origen de la cavidad obedece a procesos de erosión fluvial y circulación forzada de corrientes de agua evidenciada en la apariencia rosariforme en las paredes interiores de la estructura. Las geoformas se encuentran en su mayoría en la entrada de la cavidad en variedades como estalactitas, coladas, dientes de sierra y gours. El componente biofísico es el aspecto diferenciador de la caverna, constituido por una buena dinámica de ventilación, presencia de agua de forma permanente, alta humedad y variedad de fauna, entre los que se destacan taxas como Chiroptera, Araneae, Anura, Lepidoptera, Blattodea, Rodentia, Oligochaeta, Dermapteros, Siluriforme y Decápodos. Conclusiones: Litologicamente la cavidad se encuentra enmarcada dentro de rocas caliza con alto contenido de materia orgánica con variación en su color superficial pertenecientes a la Formación La Luna, la poca humedad de la cavidad en su entrada y alto contenido de carbonato favoreció la formación de gran cantidad de espeleotemas. Las observaciones al interior y estudios de la corriente de agua evidenciaron que las condiciones biofísicas del lugar son aptas para albergar una rica diversidad de fauna.

Palabras clave: Biología, Caverna, Espeleología, Espeleogénesis, Paisajes kársticos, Roca caliza.
Abstract
Objective: This study aims to characterize the most important aspects of the Devil's cave, including biophysical, geological, speleological and morphological components. Methods: A descriptive research from observations in situ, specific technical work of topography, geology, speleology and biology, along with study of related literature has been conducted. 
No. 2

Julio - Diciembre 2015 ISSN 0122-820X E-ISSN 2422-5053

Results: The methods used allowed us to learn about the natural richness inside the cave, with outstanding karst landforms (speleothems), presence of fish, crustaceans and other organisms that might be endemic. The information obtained indicates that the origin of the cavity is due to fluvial erosion processes and forced circulation of streams, evidenced in its shaped rosary surface on the interior walls of the structure. Landforms are mostly at the entrance of the cavity in varieties like Stalactites, Castings, Sandsicles and Gours. The biophysical component is the most distinctive aspect of the cave, consisting in a good dynamic of ventilation, permanent presence of water, high humidity and variety of wildlife, including taxa as Chiroptera, Araneae, Anura, Lepidoptera, Blattodea stand, Rodents, Oligochaeta, Dermaptera, siluriform and Decapods. Conclusions: Lithologically, the cavity is framed in limestone rocks rich in organic matter with variation in surface color belonging to the La Luna Formation, the low humidity of the cavity at its entrance and high carbonate content favored the formation of large numbers of speleothems. The inside observations and studies on the water stream showed that biophysical conditions of this place are suitable to house a rich diversity of wildlife.

Keywords: Biologia, Caverna, Espeleologia, Espeleogênese, Paisagens Cársicas, Rocha Calcária.

\section{Resumo}

Objetivo: Este estudo teve como objetivo caracterizar os aspectos mais relevantes da "Caverna do Diabo", entre os que se incluem o componente biofísico, geológico, espeleológico e morfológico. Metodologia: Realizou-se uma pesquisa descritiva a partir de observações in situ, trabalhos técnicos específicos de topografia, geologia, espeleologia e biologia, acompanhado de análise de literatura relacionada. Resultados: Os métodos utilizados permitiram conhecer a riqueza natural no interior da caverna, onde sobressaem relevo cárstico (espeleotemas), presença de peixes, crustáceos, e outros organismos que poderiam ser endémicos. A informação obtida indica que a origem da cavidade obedece a processos de erosão fluvial e circulação forçada de correntes de agua, evidenciada na aparência rosariforme nas paredes interiores da estrutura. Os relevos se encontram em sua maioria na entrada da cavidade em variedades como estalactites, coladas, dentes de serra e gours. O componente biofísico é o aspecto diferenciador da caverna, constituído por uma boa dinâmica de ventilação, presencia de agua de forma permanente, alta umidade e variedade de fauna, entre os que se destacam taxas como Quiróptera, Araneae, Anura, Lepidóptera, Blattodea, Rodentia, Oligochaeta, Dermaptera, Siluriforme e Decápodes. Conclusões: Litologicamente a cavidade se encontra emoldurada dentro de rochas de calcário com alto conteúdo de matéria orgânica com variação em sua cor superficial pertencentes à Formação "A Lua", a pouca umidade da cavidade em sua entrada e alto conteúdo de carbonato favoreceu a formação de grande quantidade de espeleotemas. As observações ao interior e estudos da corrente de agua evidenciaram que as condições biofísicas do local são aptas para ter uma rica diversidade de fauna.

Palavras-chave: Biologia, Caverna, Espeleologia, Espeleogênese, Paisagens Cársicas, Rocha Calcária. 


\section{Introducción}

La Espeleología es un campo de estudio interesante y prometedor en nuestro país que combina la ciencia, la investigación, el deporte y la aventura. Está tomando relevante importancia y cada vez es más conocida debido a los trabajos investigativos realizadas por diferentes entidades nacionales e investigadores particulares y a la protección y conservación del patrimonio geológico, en la caverna del Diablo no se reportan estudios previos. Con este proyecto se busca aportar información inédita del campo Bioespeleológico al sector académico local y nacional, seguir contribuyendo en la exploración y conocimiento de los recursos geológicos de nuestra región y divulgarlos en los espacios y entidades científicas apropiados.

En este artículo se consignan los resultados investigativos efectuados en la caverna del Diablo, en el municipio de Becerril, departamento del Cesar, producto de arduas jornadas de observación, trabajos técnicos de geología y topografía, tomas de muestras de agua y rocas, inventario espeleológico, donde se describen todas las geoformas presentes al interior de esta estructura subterránea; y un inventario biológico, en el que se describen la mayoría de organismos vivos observados, destacándose algunas especies troglobias, identificadas a partir de rasgos morfológicos troglomórficos.

\section{Localización}

El municipio de Becerril se encuentra en la región caribe colombiana, en la subregión norte del departamento del Cesar, su territorio se encuentra inmerso en dos eco regiones las cuales son la eco región Valle del Río Cesar y Serranía del Perijá. [1].

La caverna del Diablo se encuentra ubicada en la vereda El Roncón, en el extremo oriente municipal, en la Serranía del Perijá, extensión de la cordillera oriental de Colombia (Figura $1)$.

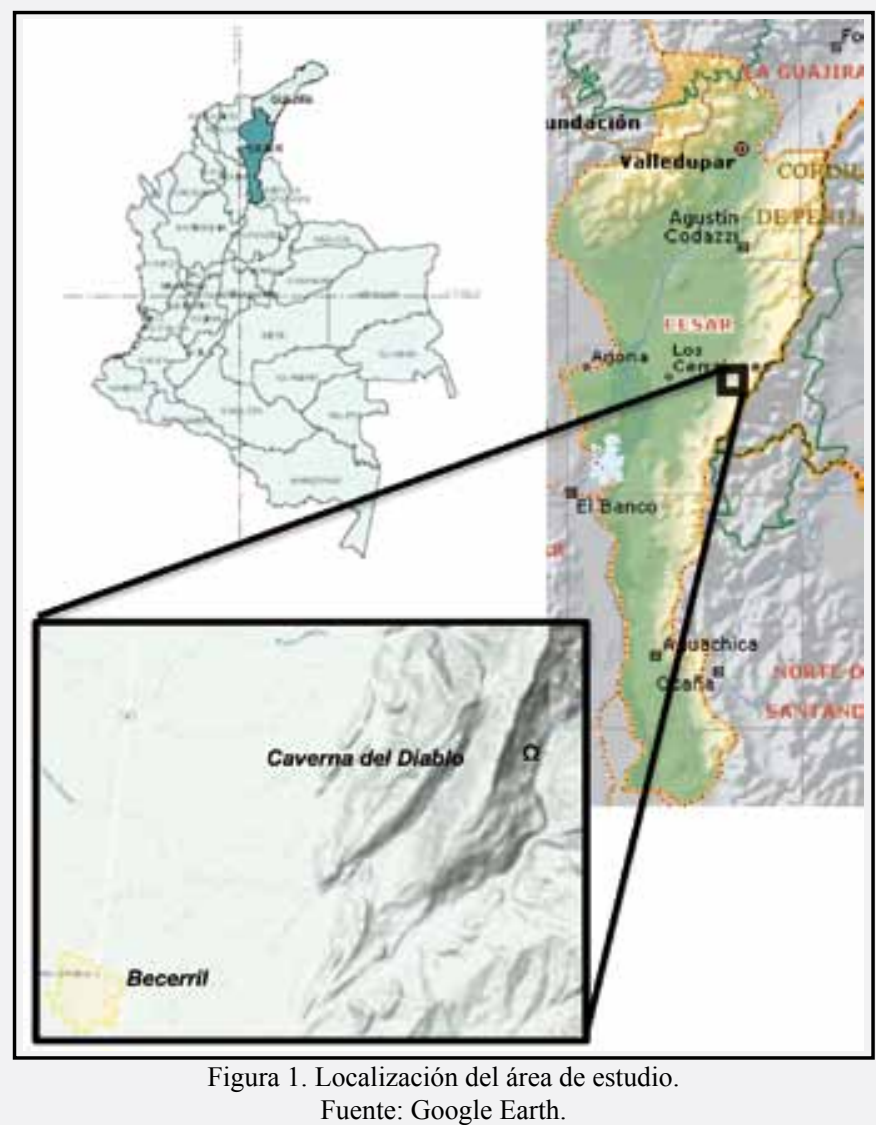




\section{Materiales y métodos}

En el inicio del trabajo investigativo se realizaron recorridos por el área de estudio para identificar y referenciar los aspectos más importantes, entre estos, los afloramientos y unidades geológicas, las estructuras espeleológicas, los puntos topográficos claves y hallazgos biofísicos y antropológicos significativos. Posteriormente se planearon los trabajos prácticos y se ejecutaron como se describe a continuación.

\section{Trabajo de Campo}

Para la ejecución de las actividades de campo se tomó como referencia la metodología empleada en el Estudio Geoespeleológico de La Caverna Sabana de León y Cueva Coco Loco En El Municipio de Manaure, Cesar [2], se hicieron observaciones in situ, inventario de los elementos relevantes, levantamientos topográficos, geológicos y espeleológicos.

Levantamiento Topográfico: las condiciones morfológicas y de acceso a la cavidad llevaron a realizar mediciones internas con métodos básicos [3], con el empleo de cintas métricas y brújulas se logró hacer el levantamiento de toda la cavidad y generar un plano bidimensional de la misma.

Se tomaron coordenadas de los sitios más relevantes con GPS de mano Garmin Etrex 30, principalmente de la entrada de la caverna con el fin de hacer su referenciación en planos y mapas digitales de escala 1:100.000 [2]. Esta información fue utilizada para identificar las unidades lito estratigráficas asociadas al área de estudio con verificación de la cartografía en el mapa geológico del Cesar de escala $1: 100.000[4],[5]$.

Toma de Muestras: Se tomaron muestras de agua de la corriente que se encuentra al interior de la caverna, de acuerdo a la Norma Técnica Colombiana ISO 5667-1 [6], y las recomendaciones dadas por el Instituto
Geográfico Agustín Codazzi para análisis físico químico [7], (Figura 2).

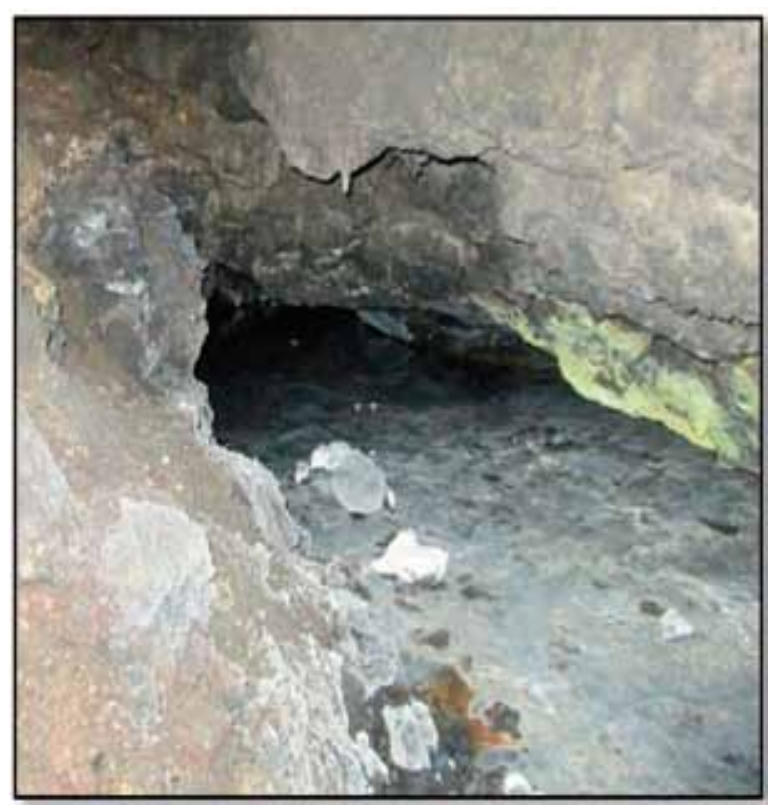

Figura 2. Afloramiento de la corriente de agua al final de la caverna con evidente presencia de azufre. Fuente: Frederick Vides-Navarro.

La roca observada al interior de la cavidad presenta un cambio físico, debido a esto, se tomaron varias muestras de roca para análisis petrográfico en los laboratorios de petrografía de la Universidad Nacional, Medellín. Las muestras recolectadas en campo fueron cortadas y pulidas para la preparación y caracterización petrográfica basada en microscopia de luz transmitida obtenida en un microscopio marca Olympus equipado con cámara fotográfica para el análisis de sección delgada.

Levantamiento Espeleológico: Es una de las actividades más arduas y detalladas del proceso, consiste en tomar las dimensiones de cada espeleotema y referenciar su ubicación respecto a la entrada de la cavidad. Además se hace una descripción morfológica de las geoformas [8], tomando detalles del aspecto físico e interpretando los procesos geoquímico que los originaron.

Geología Local: Se realiza a partir de 
observaciones en campo, describiendo las unidades lito estratigráficas presentes al interior y exterior de la caverna, se identifican diaclasas, fallas y cualquier aspecto geológico relevante, se complementa con el análisis e interpretación de los resultados de estudios petrográficos realizados en laboratorio.

Geología Regional: Se obtiene de la georeferenciación de la caverna en mapas geológicos regionales (Figura 3) y en herramientas cartográficas digitales, se complementa con el análisis e interpretación de la literatura geológica existente para definir la relación e influencia de las formaciones y estructuras geológicas cercanas a la cavidad.

Caracterización Biofísica: Se basa en observaciones realizadas In Situ sin toma de muestras faunística para tomar datos reales de las condiciones biofísicas del lugar y llevar a cabo un análisis preliminar utilizando literatura apropiada. Se hace una descripción preliminar de fauna en su interior para registrar un precedente del lugar de forma parcial y aproximada. También se lleva a cabo una descripción biofísica del área exterior de la caverna del Diablo [1], siendo el ecosistema natural próximo. Algunos datos e información del medio ambiente se obtienen de las estaciones de control y monitoreo ambiental de la zona.

\section{Resultados y análisis}

La caverna del Diablo se encuentra en las planicies de inundación de la Serranía del Perijá a una altura de 254 m.s.n.m, inmerso en la Formación La Luna, que geológicamente comprende una sucesión alternante de lutitas negras carbonosas, limolitas, arcillolitas, calizas negras, bituminosas, capas delgadas de chert y arenisca carbonatadas [4], (Figura $3)$.

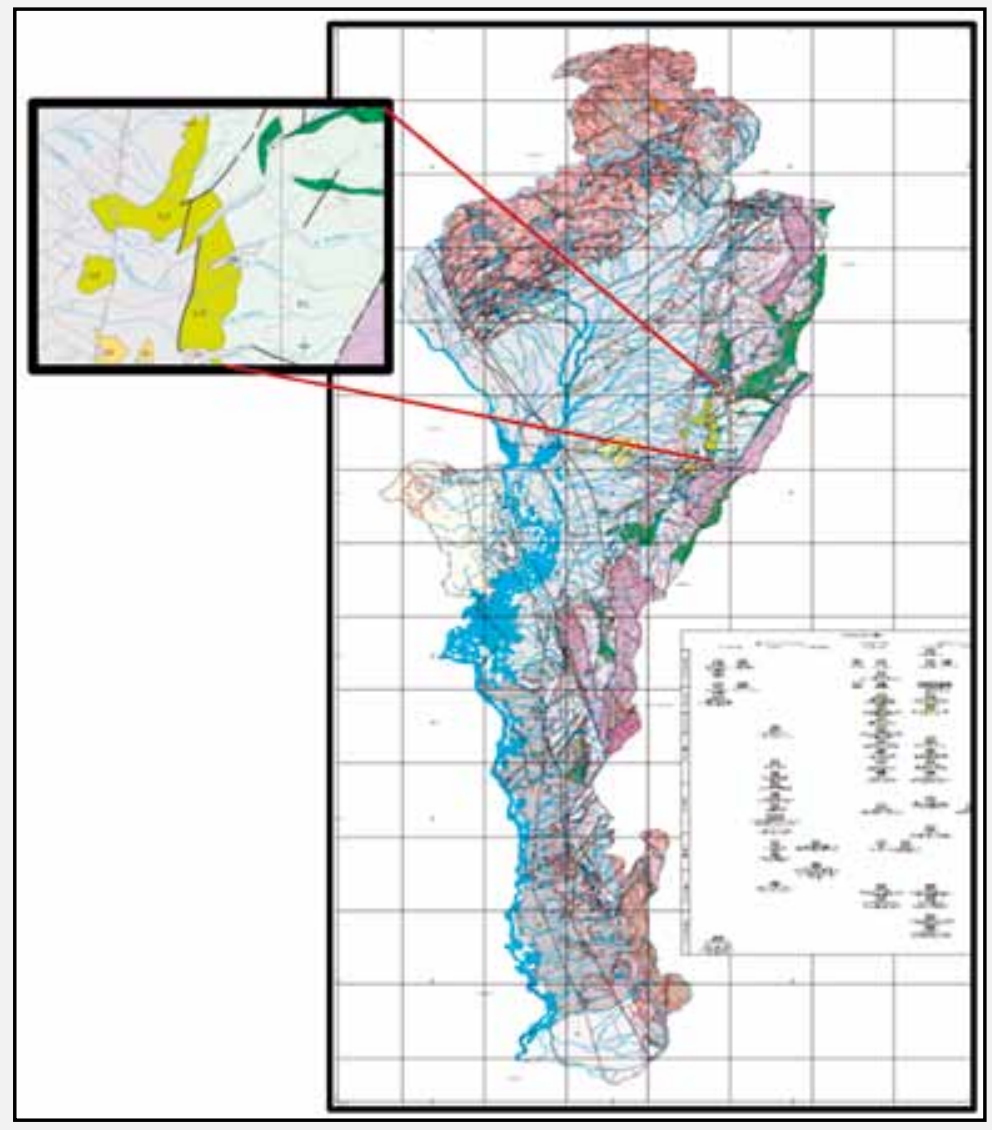

Figura 3. Ubicación de la caverna en el Mapa Geológico del Cesar, Escala 1:100.000.

Fuente: Ingeominas, Arias \& Morales, 1999. 
La cavidad se encuentra a la izquierda de la quebrada El Roncón aguas abajo, rodeada comúnmente de afloramientos, de roca básicamente constituida por calizas grises a cremas, con estratificación marcada y altamente fracturada y diaclasada.

\subsection{Morfología y Formación}

A partir de la interpretación Petrográfica de las secciones delgadas realizadas en laboratorio, evidencian que la secuencia estratigráfica en este sector de la Formación La Luna, muy probablemente se depositó en un ambiente marino profundo a costero debido a la presencia de cuarzo cristalino $\mathrm{y}$ minerales pesados que pueden tener un origen detrítico continental, la orientación preferencial de los minerales y fracturas perpendiculares a la exfoliación observables, rellenas de carbonatos, indican que la cuenca estuvo sometidos a esfuerzos tensionales que causaron el levantamiento de la misma y fluctuaciones, pasando de un ambiente marino profundo a uno poco profundo o somero tal vez restringido por la presencia de óxidos de hierro, ocasionando que la cuenca tuvieran un mayor aporte continental.

La entrada de la cavidad es una muestra clara de la irregularidad morfológica que predomina, su forma alargada tiene 7 metros de longitud y una altura máxima de $1.30 \mathrm{~m}$ (Figura 4). En su galería principal tiene una longitud de $155 \mathrm{~m}$. en los que predomina la irregularidad en cuanto a su forma. Todas las galerías presentan dimensiones diferentes que pueden ir desde $1 \mathrm{~m}$. de altura hasta los $6 \mathrm{~m}$, y $1.20 \mathrm{~m}$. de ancho hasta los $15 \mathrm{~m}$. Presenta decenas de pequeños túneles posiblemente interconectados y con acceso al exterior que permiten el ingreso a animales, agua y oxigeno favoreciendo la dinámica de ventilación de la cavidad.

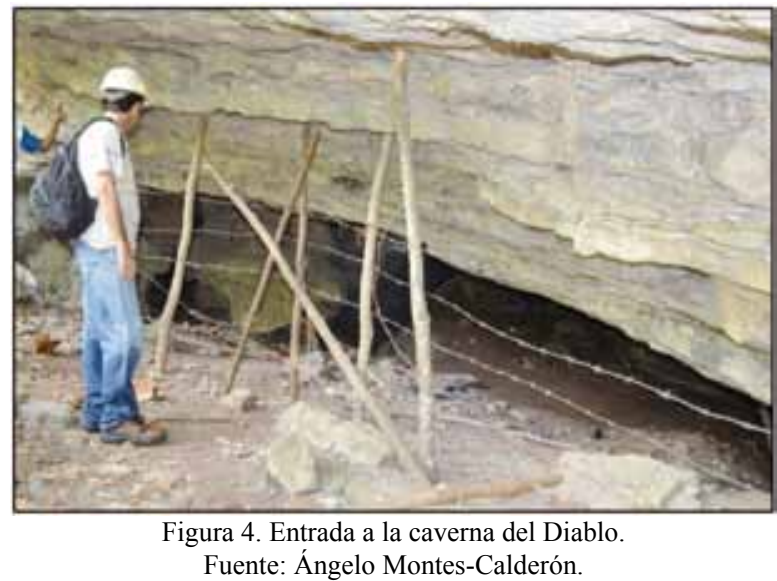

Al interior, su morfología es rosariforme, visiblemente marcada por influencia del agua. Las marcas de corrientes acuáticas en las paredes evidencian diferentes momentos de inundación (Figura 5) o transiciones de profundidad a poca profundidad o somera, quizás por la influencia de esfuerzos tensionales o movimientos tectónicos que produjera un levantamiento de la zona.

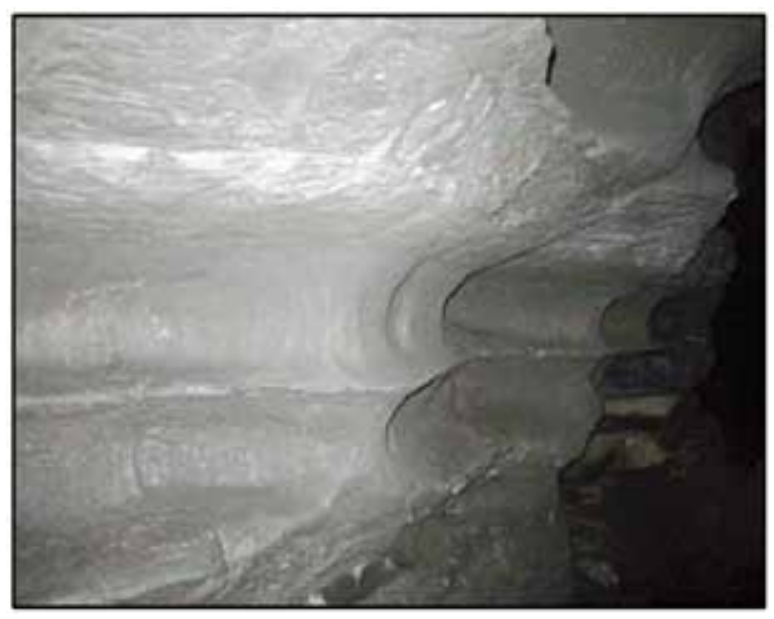

Figura 5. Pared al interior de la cavidad con marcas que evidencian diferentes momentos de inundación. Fuente: Frederick Vides-Navarro.

El área donde se encuentra la Formación La Luna se caracteriza por ser influenciada por aguas que se infiltran a través de las fracturas de las rocas calizas y se mezclan con los óxidos y sulfuros como pirita que la disuelven y producen el olor a azufre, razón a la que se debe el nombre de la caverna. La corriente de agua al interior de la cavidad 
permite establecer alguna relación con su origen, pudiendo esta ser la principal agente de los procesos de disolución de la roca [9], teniendo en cuenta que su flujo es continuo y aumenta de acuerdo a los niveles de lluvia de cada época.

Basándose en las mediciones realizadas al inicio de la investigación y utilizando herramientas cartográficas digitales, se logró generar un plano bidimensional de la caverna del Diablo (Figura 6).

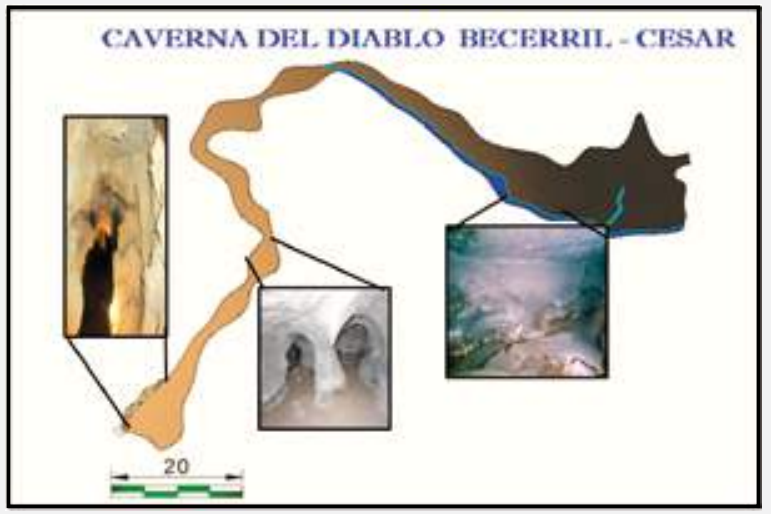

Figura 6. Plano bidimensional de la caverna del Diablo. Fuente: Frederick Vides-Navarro.

\subsection{Espeleología}

En la Caverna del Diablo, los espeleotemas se encuentran restringidos casi en su totalidad a los primeros 30 metros, donde las condiciones geológicas, físicas y químicas son apropiadas. En un pequeño pasaje paralelo a la galería principal (Figura 7), denominado túnel espeleológico, se encontraron espeleotemas de diferente forma y tamaño, las variedades más comunes que se registraron son estalactitas, coladas, y dientes de sierra [8].

\subsubsection{Espeleotemas Cenitales}

Son una de las variedades más comunes en las cavernas, son el producto de un proceso de disolución que se forma en el techo de una cavidad, y su variedad obedece a la influencia del viento, ritmo de goteo y otros.

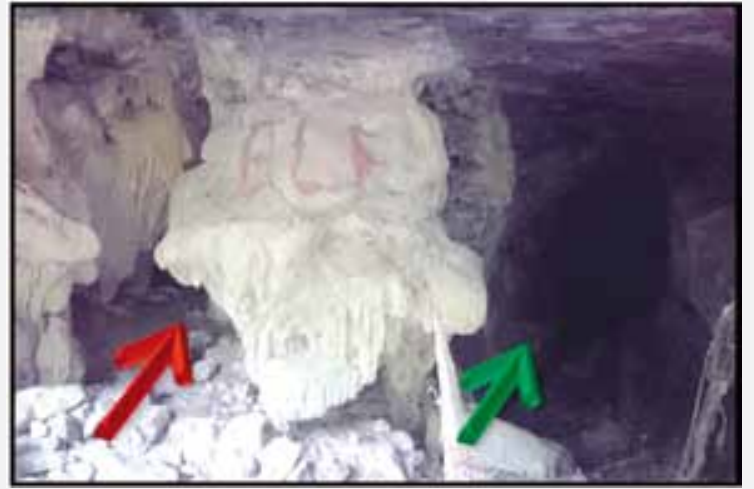

Figura 7. A la izquierda entrada del túnel espeleológico. A la derecha galería principal de la cavidad, en el centro la colada más grande. Fuente: Frederick Vides-Navarro.

\section{Estalactitas}

Es una de las formas más comunes dentro de la caverna del Diablo. Cubren gran parte del techo del túnel espeleológico, (Figura 8, Figura 9).

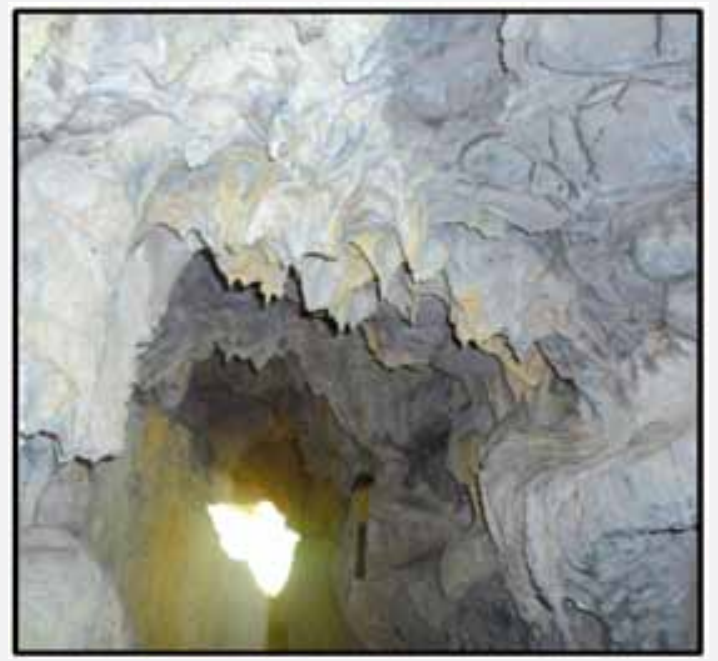

Figura 8. Estalactitas (Banderas) presentan color entre blanco y amarillo pálido.

Fuente: Frederick Vides-Navarro.

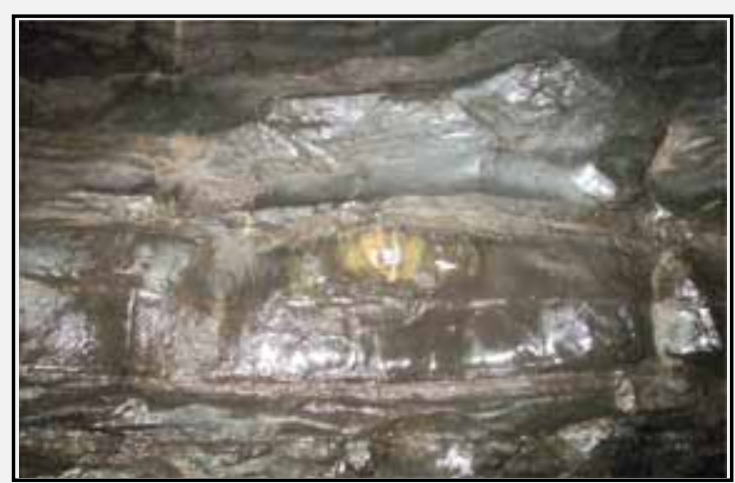

Figura 9. Estalactita ubicada en la cámara final de la caverna, se encuentra activa y presenta poco contenido de carbonato, tiene 12 $\mathrm{cm}$ de diámetro y $9 \mathrm{~cm}$ de longitud Fuente: José María Cantillo. 
No. 2

Julio - Diciembre 2015 ISSN 0122-820X E-ISSN 2422-5053

PP: 93-104

\subsubsection{Espeleotemas Pavimentarios}

Estos espeleotemas suelen ubicarse en el suelo, debajo de las estalactitas. Su formación y variedad depende de la composición del suelo al que cae y de variables como ritmo de goteo, altura de caída, evaporación, etc.

En la caverna del Diablo no existen espeleotemas de este tipo, el suelo en la entrada de la cavidad es muy seco y poco consolidado y algunas zonas están cubiertas con ramas y semillas. En el resto de la caverna hay zonas húmedas y vadosas, y las condiciones geoquímicas de la roca no favorecen la formación de estos espeleotemas.

\subsubsection{Espeleotemas Parietales}

Se originan cuando la disolución de la roca se produce sobre las paredes, son muy abundantes y es común encontrarlos en todas las cavernas.

\section{Dientes de Sierra}

Generalmente se desarrollan al final de las coladas parietales con distinta pendiente y también sobre bloques de diferentes tamaños, (Figura 10).

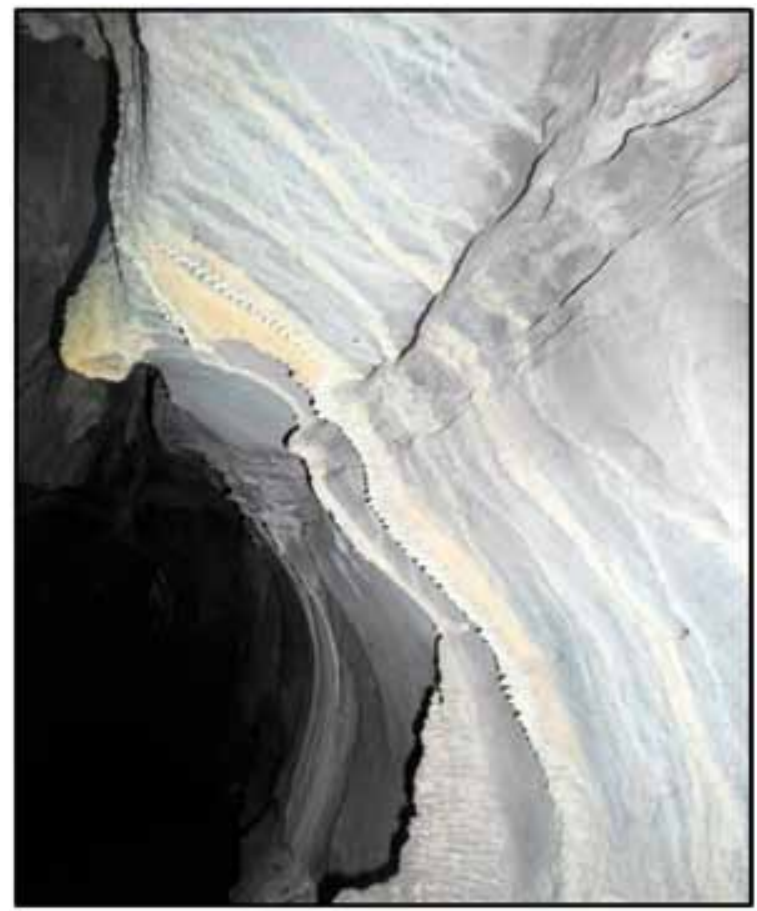

Figura 10. Diente de sierra, es el más largo de la caverna con $53 \mathrm{~cm}$ de longitud. Se encuentra al final del túnel espeleológico. Fuente: Frederick Vides-Navarro.

\section{Coladas}

Son muy frecuentes en la caverna del Diablo. Se producen cuando el agua presenta un flujo laminar sobre una determinada superficie. La colada más grande de la caverna ha crecido desde el techo, tiene $2 \mathrm{~m}$. de longitud vertical y $1.77 \mathrm{~m}$. ancho (Figura 11).

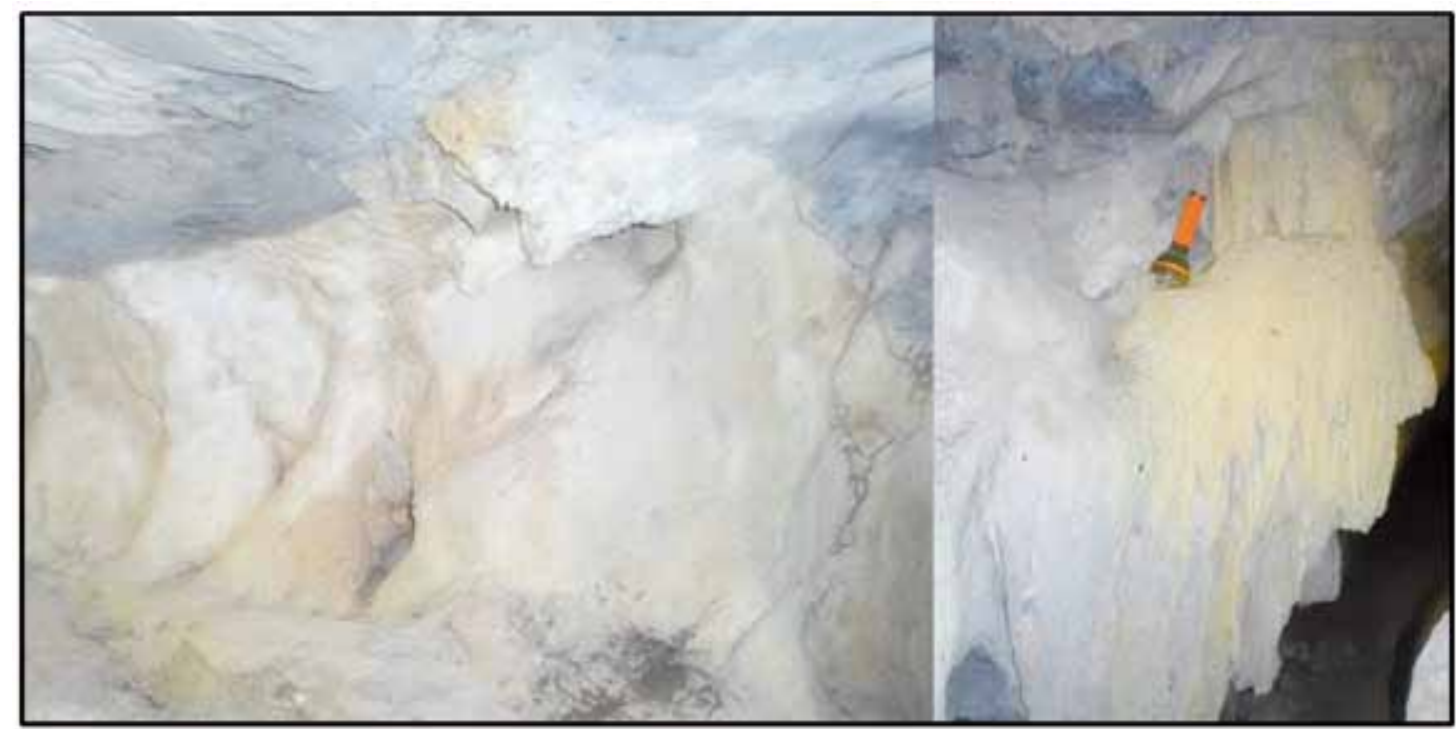

Figura 11. Coladas parietales ubicadas a la izquierda del túnel espeleológico. 
A la izquierda de la entrada de la caverna y del túnel espeleológico, los primeros metros se encuentran cubiertos por coladas que crecieron desde las paredes alcanzando $5 \mathrm{~m}$ de longitud horizontal y con alturas que van desde $66 \mathrm{~cm}$ hasta $1.50 \mathrm{~m}$. en la parte más alta.

\section{Gours}

Son una especie de diques que se forman sobre una pendiente por lo que circula un flujo laminar de agua o en el borde de charcos de agua poco profundos. Se orientan en ángulo recto con respecto a la dirección del flujo de agua. Su génesis, requiere un grado determinado de pendiente, al menos un flujo laminar de agua semi continuo y la existencia de irregularidades en el techo por lo que se desplaza el agua. La tensión superficial del agua causa la cristalización de la calcita en la interface de agua/aire/roca en algunos casos se puede encontrar a nivel parietal. Se forman debido al goteo continuo de agua desde el techo, dando como resultado la formación de pequeños cráteres en la superficie de la roca gracias al proceso kárstico que actúa sobre ella, (Figura 12).

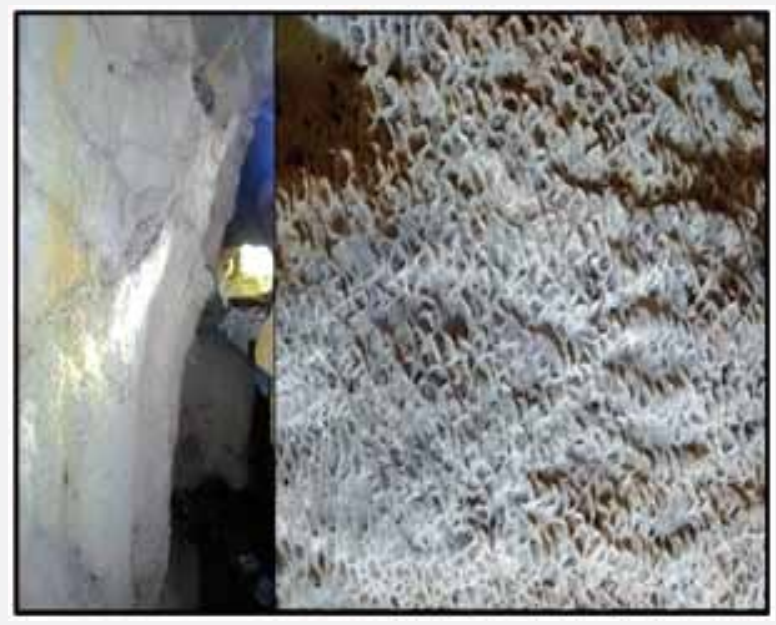

Figura 12. A la izquierda gours parietal ubicado en el túnel espeleológico y a la derecha la misma imagen ampliada donde se observa la forma característica de este espeleotema. Fuente: Giovanni Fernández-Cuello.

Existe un espeleotema en los primeros metros de la cavidad que parece haber sufrido desgaste, perdida de color y deshidratación a causa del viento y la resequedad del área, sufriendo algún proceso de meteorización físico-química, (Figura 13).

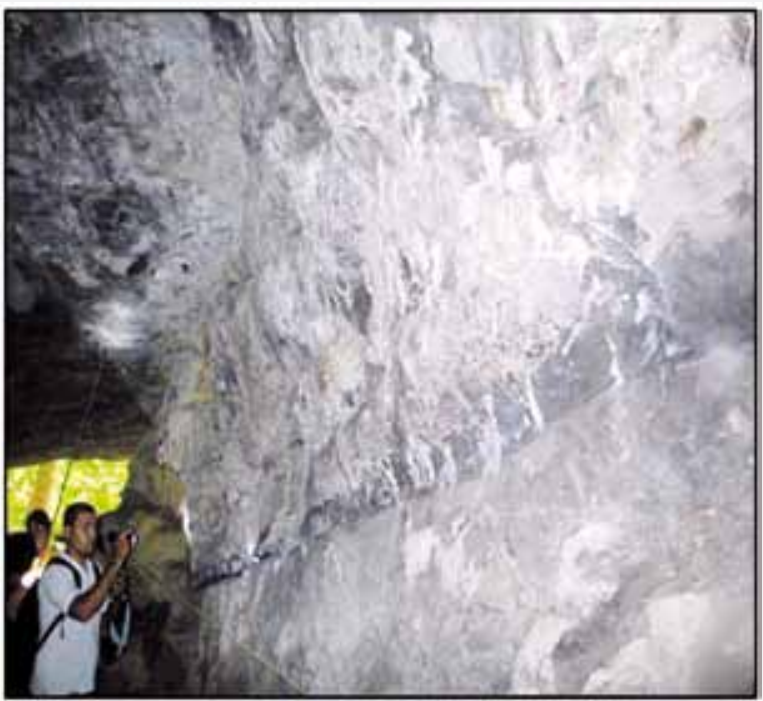

Figura 13. Pared en la entrada de la caverna con espeleotemas meteorizados, tiene $8.9 \mathrm{~m}$. horizontales y $1.5 \mathrm{~m}$. de altura. Fuente: Antonio Rudas.

\subsubsection{Caracterización Biofísica}

La caverna presenta en su interior una biodiversidad sorprendente. Esto se debe a las buenas condiciones biofísicas del lugar como su humedad, que oscila entre $75 \%$ y $85 \%$; niveles de oxígeno aptos para humanos, gracias a las decenas de pequeños túneles que conectan la cavidad con la superficie favoreciendo la dinámica de ventilación; presencia de agua de forma continua, con 2 puntos de afloramiento al final de la caverna variando su caudal de acuerdo a la época del año, siendo Mayo y Septiembre los meses con mayores niveles de precipitación en la zona (Estación Socomba, municipio de Becerril), alcanzando 370 y 400 milímetros de precipitación respectivamente; y abundante cantidad de guano, que constituye una importante fuente de energía en estos hábitats, acumulado por mucho tiempo a partir del excremento generado por los murciélagos principalmente.
ISSN 0122-820X

E-ISSN 2422-5053

PP: 93-104 
El agua está presente de forma continua en la caverna por más de 30 metros, poseen un pH neutro, coloración amarrillo pálido, su conductividad la enmarca entre el rango de excesivamente contaminada, parámetro que tal vez haga alusión al hecho de que Por gravedad las aguas de percolación arrastran nutrientes; diversos tipos de materiales orgánicos a las aguas. Los contenidos mineralógicos de hierro, plata, sulfatos, nitratos, cloruros y nitritos son muy bajos no generando riesgos de toxicidad, lo que confirman que estas aguas son propicias para albergar biota y ecosistemas propios de ambientes cársticos subterráneos.

En conjunto la fauna terrestre es predominantemente troglofila, la acuática tiene mayor representación de organismos troglobios, pero por su biomasa los individuos trogloxenos llegan a ser considerable.

Se logró realizar un somero inventario con los taxas encontrados:

Chiroptera: son el grupo más numeroso observado y son conspicuas las siguientes familias Emballonuridae, Noctilionidae, Mormoopidae, Phyllostomidae y Vespertilionidae. Esta última ubicada cerca de la entrada de la caverna. La presencia de estos troglóxenos aporta materiales que se acumulan en el ambiente intermedio en forma de guano y semillas, incluso sus cadáveres y restos también hace parte de los nutrientes que sostiene a la mayoría de los taxas de invertebrados que hacen parte del mismo biotopo.

Araneae: Constituyen uno de los más importantes grupos de arácnidos. Las arañas cavernícolas observadas poseen formas intermedias entre la vida epígea y la hipógea, estando presentes mayormente cerca a la entrada de la cavidad. Algunas de ellas son troglófilas y una troglobia (depigmentada, estilizada y anoftalma).
Anura: Este orden estaba representado por las familia Buffonidae y Hylidae, anfibios troglóxenos cuya presencia se debe seguramente a que encontraba suficiente alimento entre los invertebrados de la cueva.

Lepidoptera: Se observaron mariposas adheridas en las paredes de la cueva y también se encontraron en estados larvarios.

Blattodea: Las cucarachas fue uno de los grupos de insectos observados con mayor frecuencia.

Rodentia: Se tomó en cuenta la presencia de un roedor de gran tamaño deambulando por las orillas de la caverna.

Oligochaeta: En los depósitos de arena y bordes con alta presencia de humedad (ambiente profundo) fueron encontrados anélidos oligoquetos en bajo número.

Dermapteros: Se observaron algunos individuos con características epígeas y otras hipógeas con decoloración pronunciada de su cuerpo.

Siluriforme: $\mathrm{Se}$ registraron peces (posiblemente pertenecientes a la familia Pimelodidae) comúnmente llamados en la región como barbul, con un tamaño promedio oscilando desde 2 hasta $9 \mathrm{~cm}$ aproximadamente y con evidente pérdida de la pigmentación que permite clasificarlo como una especie troglobio, que posee ojos de talla muy reducida, probablemente no funcionales.

Decápodos: Observación de un crustáceo, cangrejo con particularidades pertenecientes a la Familia Pseudothelphusidae además no presenta pigmentación y otras características que lo enmarcan como una especie troglobia, (Figura 14). 


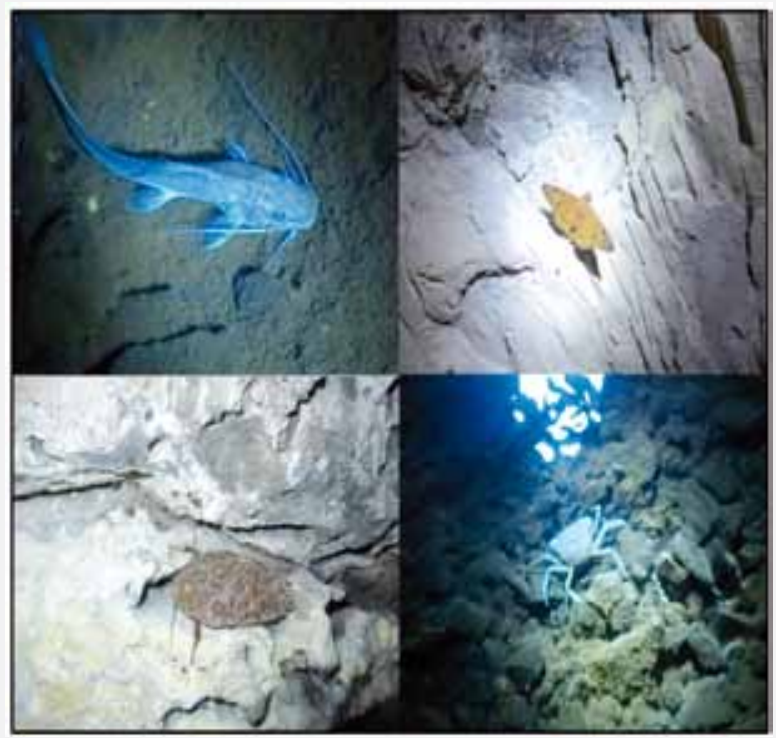

Figura 14. Algunas de las especies de animales encontradas en la caverna.

Fuente: José María Cantillo.

\section{Conclusiones}

Al interior de la caverna del Diablo se identifica un cambio físico en la roca, en la entrada se encuentran calizas con una coloración cremas grisáceas micríticas sobre la cual se encuentra la formación de la mayoría de espeleotemas presentes, a partir de los $25 \mathrm{~m}$. la composición mineralógica de la roca caliza cambia, se torna más oscura, bituminosa con alto contenido de materia orgánica, con presencia de algunas venas de carbonatos, esta variación composicional unidos a parámetros como temperatura y ventilación, hacen que la generación de espeleotemas esté restringida a los primeros metros de la cavidad, donde los más comunes son las estalactitas, coladas y dientes de sierra.

La cavidad presenta en su interior una morfología rosariforme y se aprecia claramente que su origen obedece a un proceso de disolución, circulación forzada de corrientes de agua y erosión fluvial. El agua que existe en su interior se infiltra por conductos del sistema acuífero Formación La Luna, fracturas y planos de estratificación de las rocas. Esta posee un $\mathrm{pH}$ neutro, coloración amarrillo pálido con un valor de
20 UPC, indicador de presencia de pocos materiales contaminantes, no obstante por los valores arrojados en la conductividad estas aguas se enmarcan entre el rango de excesivamente contaminadas, son aguas algo turbias, con bajo contenido de solidos suspendidos; los contenidos mineralógicos de hierro, plata, sulfatos, nitratos, cloruros y nitritos son muy bajos no generando riesgos de toxicidad, indicando que la corriente de agua es propicia para albergar biota $\mathrm{y}$ organismos propios de estos ambientes cársticos subterráneos.

En el interior de esta estructura subterránea existen evidencias de que la cavidad ha servido por mucho tiempo como refugio para diversos animales. Además es un complejo ecosistema subterráneo que no solo es un refugio con microclima más húmedo que los ambientes epigeos circundantes, sino que es el hábitat permanente $\mathrm{y}$ exclusivo para invertebrados que podrían haber evolucionado allí, independientemente de la fauna epigea. Es decir que la caverna del Diablo es un excelente modelo para estudios científicos de biología subterránea.

\section{Agradecimientos}

Los autores desean expresar sus agradecimientos a:

Colciencias, a la Fundación Universitaria del Área Andina por el apoyo financiero en la realización de esta investigación, a La Cruz Roja y Secretaría de Cultura de Becerril, al Ingeniero Antonio Rudas Muñoz, al Docente José María Cantillo Lozano, a la Bióloga Laura Rojas y al Semillero de Investigación Geológico Minero (SIGEM) de la Fundación Universitaria del Área Andina, por su acompañamiento, apoyo logístico y asesoría.

\section{Referencias}

[1] A. Rudas, "Caracterización Biofísica de la Caverna del Diablo en el municipio 
[2] H. Pavajeau, A. Carrillo y E. Rojas,

[3] A. Salazar. Prácticas de topografía.

[4] A. Arias, C. Morales. Evaluación del

[5] Ingeominas. Mapa geológico

[6] Norma Técnica Colombiana ISO 5667-

[7] Instituto Geográfico Agustín Codazzi.

[8] Sociedad Espeleológica Colombiana

[9] C. Benedetto, G. Redonte. Introducción "Estudio geoespeleológico de la Caverna Sabana de León y Cueva Coco Loco en el municipio de Manaure, Serranía de Perijá, Cesar". Trabajo de grado ingeniería de minas. Valledupar: Fundación Universitaria del Área Andina. Facultad de ingeniería de minas, p. 49, 2011. México: Universidad Autónoma Nacional de México, 1987. agua subterránea en el Departamento del Cesar, Informe Geológico. Santa Fe de Bogotá: Corpocesar - Ingeominas, p.104, 1994. generalizado del Departamento del Cesar, Memoria explicativa. Santa Fe de Bogotá: Ingeominas, 1999. 1. Gestión ambiental. Calidad del agua. Muestreo. Directrices para el diseño de programas de muestreo. Santa $\mathrm{Fe}$ de Bogotá: Icontec, 1995. Análisis de aguas. [Online]. Disponible en: http://www.igac.gov.co/wps/portal/ (ESPELEOCOL). Grupo de Investigación Geomorfológica y Procesos Fluviales. Santa Fe de Bogotá: Universidad Nacional de Colombia, 2010. a la Espeleología. Malargüe: Escuela

Argentina de Espeleología, p. 19, 2005.

de Becerril, departamento del Cesar" igac/raiz/iniciohome/tramites.
[10] D. Culver, T. Pipan. Biology of Caves and Other Subterranean Habitats. Oxford: Oxford University Press, 2009. 